\title{
Influence of sprint duration during minimal volume exercise on aerobic capacity and affect
}


Matthew Haines $^{\mathrm{a}^{*}}$, David Broom ${ }^{\mathrm{b}}$, John Stephenson ${ }^{\mathrm{a}}$ and Warren Gillibrand ${ }^{\mathrm{a}}$

a School of Human and Health Sciences, University of Huddersfield, Huddersfield, United Kingdom; ${ }^{b}$ Centre for Sports, Exercise and Life Sciences, Coventry University, Coventry, United Kingdom

\section{* Corresponding author:}

Dr Matthew Haines

University of Huddersfield

School of Human and Health Sciences

Queensgate campus

Huddersfield, HD1 3DH, United Kingdom

Email address: M.Haines@hud.ac.uk 


\begin{abstract}
The aims of this study were to investigate the influence of reduced-exertion, high-intensity interval training (REHIT), comparing a novel shortened-sprint protocol (SSREHIT) against a traditional protocol (TREHIT), on perceptual responses and to determine if changes in peak oxygen uptake $\left(\dot{V} \mathrm{O}_{2 \text { peak }}\right)$ are attenuated with shorter sprints. Twenty-four healthy men undertook 15 sessions of SSREHIT or TREHIT. $\dot{V} \mathrm{O}_{2 \text { peak }}$ was determined at baseline and after completion of each exercise condition. Affective (pleasure-displeasure) responses and perceived exertion were assessed during exercise to capture peak responses. Enjoyment was recorded 5-min after cessation of exercise. Compared to baseline, $\dot{V} \mathrm{O}_{2 \text { peak }}$ increased in both groups (6\% for SSREHIT $[d=-0.36]$ and $9 \%$ for TREHIT [ $d=-0.53], \mathrm{p}=0.01)$. Affective responses were more favourable for SSREHIT $(\mathrm{p}=0.001, d$ $=1.62$ ), but both protocols avoided large negative peaks of displeasure. Peak ratings of perceived exertion were lower for SSREHIT $(\mathrm{p}=0.001, d=-1.71)$, although there were no differences in enjoyment $(d=0.25)$. The results demonstrate both exercise conditions can increase $\dot{V} \mathrm{O}_{\text {2peak }}$ without overly compromising perceptual responses. Decreased sprint duration might further circumvent negative perceptual responses but might also attenuate physiological adaptations.
\end{abstract}

\title{
Key words
}

Enjoyment; Feeling; High intensity; Interval training; Oxygen uptake; Perceived exertion 


\section{Introduction}

Regular physical activity and structured exercise are preventative and therapeutic behaviours in the management of several chronic diseases [1]. Yet poor adherence to exercise programmes remains a significant challenge with perceived lack of time the most frequently cited internal barrier [2,3]. This conflicts with current best evidence guidelines for health which encourage the accumulation of relatively high volumes of exercise. For example, guidelines recommend adults should be physically active for a minimum of either $150 \mathrm{~min}$ at moderate intensity or $75 \mathrm{~min}$ at vigorous intensity per week (or a combination of both), in addition to avoiding time spent sedentary and exercising to improve muscle strength on at least two days per week [4, 5]. High-intensity interval training (HIT) might help to circumvent this problem because it includes brief periods of repeated, high-intensity or maximal 'sprints' interspersed with longer periods of recovery and is considered more time-efficient [6]. However, despite an emerging evidence base for the benefits of this type of activity, including improvements in several markers of cardiometabolic health risks [7, 8], HIT might evoke a high degree of discomfort and negative affect (displeasure) which may lead to an avoidant response and poor exercise adherence [9]. As such, research exploring the minimal amount of tolerable exercise to improve health is needed [10].

Reduced-exertion HIT (REHIT) was developed to be time-efficient, less strenuous, and more applicable to an inactive population, comprising 10-min cycling, inclusive of a warm-up and cool down, with $2 \times$ 10-20-s cycle sprints against a braking force equivalent to $7.5 \%$ of body mass [11]. Despite the lower volume of exercise, it has been shown to increase aerobic capacity (measured as peak oxygen uptake $\left.\left[\dot{V} \mathrm{O}_{2 \text { peak }}\right]\right)$ by $10-15 \%$ in sedentary but healthy men and women $[11,12]$, which is consistent with higher-volume approaches to HIT [13]. This is clinically important because $\dot{V} \mathrm{O}_{2 \text { peak }}$ exerts a powerful protective effect against cardiovascular disease and all-cause mortality [14] and might be more important than the overall volume of exercise [15]. Thus, the potential for REHIT to induce improvements in $\dot{V} \mathrm{O}_{2 \text { peak }}$ in a time-efficient manner could be valuable as part of an exercise strategy to improve public health. 
Hedonic theories of motivation have been used to oppose the likely adoption of more strenuous highervolume HIT $[9,16,17]$, but it remains unclear if REHIT could be a more tolerable approach for the general population. Research using perceived exertion has reported relatively low ratings. For example, inactive but healthy individuals [11, 12] and men with type 2 diabetes [18] rated REHIT as 'somewhat hard' to 'hard', although other research using recreationally active participants reported REHIT to be 'very hard' [19]. However, true responses to REHIT were potentially confounded in these studies because they were recorded after the cessation of exercise which is associated with a general unanimous shift in affective valence toward pleasure [20]. More recently, Songsorn et al. [21] observed that affect and exertion responses during REHIT were no more negative compared to moderate-intensity continuous training or higher volume HIT, suggesting that REHIT is not inherently associated with negative responses.

Since hedonicity is an important signal for behaviour optimisation, it is imperative to exploit the exercise dose-response relationship to maximise physiological benefit without overly compromising affective response. Indeed, this could be decisive because memory associated with affective responses is influenced in part by the moment a distinct 'peak' displeasure response is experienced [20]. During REHIT this peak response is likely to be coupled with high-intensity sprint activity which, despite minimal duration, could adversely influence retrospective evaluations of the activity. Accordingly, there is a need for more studies to examine the modifying effects of a range of REHIT training parameters. One practical approach to achieve this is to reduce the duration of the sprints. Nalçakan et al. [22] reduced the sprint duration of traditional REHIT from 20 -s to 10 -s and found it did not result in more positive affective responses or lower perceived exertion. A plausible explanation for this is that the protocols were not sufficiently dissimilar and thus resulted in a similar amount of anaerobic substrate phosphorylation. Also, perceptions were again captured retrospectively which might obfuscate responses during REHIT. It is possible that further reductions in sprint duration could attenuate negative perceptual responses for people who do not meet physical activity guidelines. Theoretically, this could be effective because rapid physiological adaptations associated with sprint cycling may be caused by rapid glycogenolysis stimulated during the initial seconds of maximal sprints [23]. 
Therefore, in the present study a novel, shortened-sprint REHIT (SSREHIT) protocol was compared to traditional REHIT (TREHIT). SSREHIT further decreases sprint duration (to 5-s) for which an acute bout has been associated with more favourable affective responses [24]. However, it is not known if SSREHIT attenuates improvements in $\dot{V} \mathrm{O}_{2 \text { peak }}$, or how affective responses change over time. Thus, the aims of this research were: 1) To investigate the influence of REHIT (SSREHIT and TREHIT) on $\dot{V} \mathrm{O}_{2 \text { peak }}$, and 2) To measure affective and perceptual responses throughout a longitudinal intervention.

\section{Materials and Methods}

\section{Participants}

Twenty-four men agreed to take part in the research and were informed of the benefits and risks prior to signing an informed consent form, before random assignment to SSREHIT ( $n=12$; age $20.9 \pm 1.4$ years; body mass, $80.7 \pm 6.3 \mathrm{~kg}$; body mass index [BMI], $25.6 \pm 2 \mathrm{~kg} \cdot \mathrm{m}^{2}$; fat mass, $16.2 \pm 5.8 \%$ ), or TREHIT ( $n=12$; age $21.4 \pm 2.4$ years; body mass, $79.6 \pm 7.6 \mathrm{~kg}$; BMI, $25.4 \pm 1.7 \mathrm{~kg} \cdot \mathrm{m}^{2}$; fat mass, 14.9 $\pm 5.5 \%)$. Participants were healthy, determined via negative responses to a medical screening questionnaire, inactive (i.e. not meeting physical activity guidelines), and were not engaged in structured endurance training or interval exercise programmes. Change in $\dot{V} \mathrm{O}_{2 \text { peak }}$ was designated the primary outcome variable. An a priori power analysis was performed using $\mathrm{G}^{*}$ Power software (version 3.1.9.2, 2017) based on an anticipated small to medium effect size (i.e. 0.3) [25], an alpha criterion of 0.05 , and power of $0.8(1-$ beta). This indicated a required sample size of 12 participants per group.

\section{Experimental procedures}

Following study familiarisation and baseline measurement of $\dot{V} \mathrm{O}_{2 \text { peak }}$, participants commenced the intervention within one week. Outcome measures were repeated within one week of completing the intervention. Participants were encouraged to continue consuming their normal diet and to maintain habitual physical activity routines throughout the study period.

Participants were asked to undertake a total of 15 sessions of SSREHIT or TREHIT, with two or three sessions per week permitted (i.e. the intervention lasted 5-7 weeks, although most participants 
completed within 5 weeks: SSREHIT, $n=10$; TREHIT, $n=9$ ). All exercise sessions were performed on a Wattbike cycle ergometer (Wattbike Pro, Nottingham, UK) with pedal resistance for the sprints set using the air and magnetic settings to create a flywheel braking force appropriate for peak power generation, as recommended by the manufacturer. Participants remained in the laboratory for 10-min post-exercise for monitoring of adverse events and were told to contact the principal investigator or seek medical help if they experienced any signs of a cardiac event or change in health circumstances at any point during the study. The study met the ethical standards of sports and exercise science research [26], with ethical approval to carry out the research obtained by the local institutional ethics panel.

An overview of both exercise conditions is presented in Fig. 1. For SSREHIT, participants performed 10-min of cycling, inclusive of a series of 5-s maximal effort sprints. Exercise intensities inbetween sprints were low $(\sim 60 \mathrm{~W})$. A warm-up (3-min at $\sim 30-60 \mathrm{~W})$ and cool down $(2-\mathrm{min}$ at $\sim 30 \mathrm{~W})$ were included within the 10-min session. TREHIT was performed as per Metcalfe et al. [11] and included 20-s sprints. SSREHIT was designed to match the total time spent completing high-intensity exercise consistent with TREHIT (i.e. $8 \times 5$-s sprints vs. $2 \times 20$-s sprints, respectively).

\section{[Figure 1 near here]}

\section{Outcome measures}

$\dot{V} \mathrm{O}_{2 \text { peak }}$ was determined using a ramp cycling test. Participants cycled on a magnetically braked ergometer (Ergomedic 839E Digital, Monark, Vansbro, Sweden) starting at $20 \mathrm{~W}$, with required power to continue cycling increased by $15 \mathrm{~W}$ every 1 -min thereafter, until volitional exhaustion. Participants respired through a face mask connected via a sample line and volume transducer to an online gas

analysis system (Metamax 3B, Cortex, Leipzig, Germany). $\dot{V} \mathrm{O}_{2 \text { peak }}$ was taken as the highest value averaged over 10 -s periods.

Affective valence was assessed using the Feeling Scale (FS) [27]. The FS uses a bipolar scale and ranges from -5 'very bad' to +5 'very good', with anchors designated for 0 ('neutral') and all odd integers inbetween. FS was recorded at 25\%,50\%, 75\%, and $100 \%$ of bout completion (Fig. 1). For both exercise conditions, values at $75 \%$ captured responses at their peak value and were subsequently used for further analyses. This is in-line with previous research using these protocols [24] and captured 
responses shortly after the end of sprint activity. The FS was explained to participants and presented using a visual cue card at each time point to ensure accurate reference to the scale. Aggregate mean FS responses for sessions 1-3, 4-7, and 8-15 were recorded for analyses. These sessions were chosen to capture responses across the intervention reflecting the incremental progression in the number of highintensity sprints per session (Fig. 1). Rating of perceived exertion (RPE) was monitored using the 15point Borg scale [28]. The scale ranges from 6 'no exertion' to 20 'maximal exertion' with anchors designated for all the odd integers inbetween. RPE was recorded at the same time as FS and analysed in the same way. Enjoyment was recorded 5-min post-exercise using the single-item, 7-point Exercise Enjoyment Scale (EES) [29]. Anchors are given at every integer, ranging from 1 'not at all' to 7 'extremely'.

\section{Statistical analyses}

Statistical analyses were carried out using IBM SPSS Statistics version 26 (IBM, Armonk, USA) with the criterion for statistical significance set at $\mathrm{p}<0.05$. Normal distribution of data and identification of outliers and extreme scores were assessed via visual inspection of histograms and box plots and using the Shapiro-Wilk test. The influence of the exercise conditions was then assessed by fitting linear models. Two-way (condition $\times$ time) mixed analysis of variance (ANOVA) was used to compare differences in $\dot{V} \mathrm{O}_{2 \text { peak }}, \mathrm{FS}, \mathrm{RPE}$, and EES. The sphericity assumption was checked using Mauchly's test with corrections (Greenhouse-Geisser when $\varepsilon<0.75$ and Hyunh-Feldt when $\varepsilon \geq 0.75$ ) applied to the degrees of freedom when this was violated. Significant main effects were considered using post-hoc Bonferroni-corrected pairwise comparisons to control for familywise error rate. Homogeneity of variance between groups was checked using Levene's test. Effect sizes were also calculated using partial eta squared $\left(\eta^{2}\right)$ statistic with the magnitude of difference considered as small $(<0.1)$, medium (0.1-0.3), or large (>0.5). Paired samples t-tests were then used to test for differences between pre- and post-intervention $\dot{V} \mathrm{O}_{2 \text { peak }}$ within each condition, using Cohen's $d$ to indicate the magnitude of difference, with differences considered as trivial $(<0.20)$, small $(0.20-0.49)$, moderate $(0.50-0.79)$, or large $(>$ $0.80)$. 


\section{Results}

All participants completed 15 exercise sessions. There were no dropouts and each participant undertook each exercise condition as allocated with outcome measures obtained from all participants. Five incidences of mild-to-moderate nausea or light headedness were reported (two for SSREHIT and three for TREHIT), and one participant vomited following TREHIT. There were no instances of syncope or musculoskeletal injuries in response to any of the exercise sessions.

ANOVA revealed a significant main effect of time for $\dot{\mathrm{O}}_{2 \text { peak }}\left(\mathrm{F}_{1,22}=30.6, \mathrm{p}=0.001, \eta^{2}=0.58\right)$. Compared to baseline, values increased by $6 \%$ for SSREHIT (pre: $46.2 \pm 7$ vs post: $48.8 \pm 6.6$ $\mathrm{ml} \cdot \mathrm{kg} \cdot \mathrm{min}^{-1}, d=-0.36$ ) and by $9 \%$ for TREHIT (pre: $44.7 \pm 6.8 \mathrm{vs}$ post: $48.7 \pm 7.7 \mathrm{ml} \cdot \mathrm{kg} \cdot \mathrm{min}^{-1}, d=-$ 0.53). However, the difference between groups and the interaction effect did not reach statistical significance. Variability in training response was large for both conditions (range: $-2.4 \%$ to $22.5 \%$ SSREHIT, and $-1.7 \%$ to $19.5 \%$ for TREHIT, Fig. 2).

\section{[Figure 2 near here]}

Comparison of perceptual responses for both conditions are shown in Fig. $\mathbf{3}$ and summarised in Table 1. ANOVA revealed a significant main effect of time for $\mathrm{FS}\left(\mathrm{F}_{2,44}=89.4, \mathrm{p}=0.001, \eta^{2}=0.80\right)$. Posthoc tests revealed FS to be significantly lower (more displeasure) during sessions 4-7 and 8-15 compared to the preceding time points $(p=0.001)$. There were significant interactions between condition and time, $\left(\mathrm{F}_{2,44}=3.2, \mathrm{p}=0.05, \eta^{2}=0.13\right)$ with steeper slopes of change evident for TREHIT. There was also a significant difference between conditions $\left(\mathrm{F}_{1,22}=13.4, \mathrm{p}=0.001, \eta^{2}=0.38\right)$, with peak FS values corresponding to 'fairly good' for SSREHIT and 'neutral' for TREHIT.

For RPE, ANOVA revealed a significant main effect of time $\left(\mathrm{F}_{2,44}=282.5, \mathrm{p}=0.001, \eta^{2}=0.93\right)$. As per affective responses, post-hoc testing revealed RPE to be significantly higher during sessions 4-7 and $8-15$ compared to the preceding time points $(p=0.001)$, in addition to significant interaction effects $\left(\mathrm{F}_{2,44}=5.5, \mathrm{p}=0.007, \eta^{2}=0.20\right)$ indicative of steeper slopes of change for TREHIT. There was also a significant difference between conditions $\left(\mathrm{F}_{1,22}=25.8, \mathrm{p}=0.001, \eta^{2}=0.54\right)$ with peak RPE responses 
reported as between 'somewhat hard' and 'hard' for SSREHIT, and between 'hard' and 'very hard' for TREHIT.

Similarly, ANOVA revealed a significant main effect of time for enjoyment $\left(\mathrm{F}_{1.43,31.37}=23.3, p=0.001\right.$, $\left.\eta^{2}=0.52\right)$ with post-hoc testing showing EES values to be significantly lower during sessions 4-7 and 8-15 ( $\mathrm{p}<0.05)$. However, there was no significant differences between conditions and no significant interaction effect.

[Table 1 near here]

[Figure 3 near here]

\section{Discussion}

The aims of this study were to investigate the influence of sprint duration during REHIT on perceptual responses, and to determine if changes in $\dot{V} \mathrm{O}_{2 \text { peak }}$ are attenuated when sprint durations are reduced from 20-s (TREHIT) to 5-s (SSREHIT). The main findings were that both exercise conditions significantly increased $\dot{V} \mathrm{O}_{2 \text { peak }}$ compared to baseline without overly compromising affective responses. For $\dot{V} \mathrm{O}_{2 \text { peak }}$, differences observed between groups did not reach statistical significance, although a trend for greater improvement was observed following TREHIT (9\% compared to $6 \%$ for SSREHIT). These increases are similar to values reported in previous research using REHIT with inactive individuals and type 2 diabetes patients $[12,18]$, but lower than research using healthy, young adults [11]. Compared to these studies, which used 18 to 24 sessions, the present study demonstrated that as few as 15 sessions can induce training adaptations. Although improvements in $\dot{V} \mathrm{O}_{2 \text { peak }}$ were not as high as those reported for higher-volume and more strenuous HIT [30], they may nevertheless be clinically important since an increase in $\dot{V} \mathrm{O}_{2 \text { peak }}$ of one metabolic equivalent task (MET, $\left.3.5 \mathrm{~mL} \cdot \mathrm{kg}^{-1} \cdot \mathrm{min}^{-1}\right)$ has been shown to confer a $12 \%$ improvement in all-cause mortality [31]. In the present study, the mean increase in $\dot{V} \mathrm{O}_{2 \text { peak }}$ was $4 \mathrm{~mL} \cdot \mathrm{kg}^{-1} \cdot \mathrm{min}^{-1}$ for TREHIT $\left(50 \%\right.$ of participants increased by $\geq 1 \mathrm{MET}$ ) and $2.8 \mathrm{~mL} \cdot \mathrm{kg}^{-1} \cdot \mathrm{min}^{-1}$ for SSREHIT ( $33 \%$ of participants increased by $\geq 1$ MET). Therefore, the focus on minimising negative perceptual responses via shortened sprints might compromise physiological training adaptation, as 
evidenced elsewhere [22]. Nevertheless, the potential for both TREHIT and SSREHIT to bring about rapid improvements in fitness in a time-efficient manner is notable.

Considerable heterogeneity in training response was observed for $\dot{V} \mathrm{O}_{2 \text { peak }}$. These findings support similar inter-individual variation reported following REHIT [12] and higher-volume HIT [32] and emphasise the importance of looking beyond typical responses when interpreting exercise intervention outcomes. The notion of 'responders' and 'non-responders' in relation to $\dot{V} \mathrm{O}_{2 \text { peak }}$ has been established for continuous aerobic exercise [33] and could also apply to REHIT. This should be considered when attempting to translate research into real-world practice, especially since REHIT studies [e.g. 11, 12, $18,21]$ have used small samples making mean results susceptible to the influence of individual variation. Attempts have been made to identify discrete genetic biomarkers to predict adaptations to endurance training [34] and it remains to be established whether this applies to intermittent type exercise. Preliminary research shows that individual patterns of response differ between endurance training and HIT, suggesting that non-response to endurance training might not necessarily predict response to HIT [35].

Confirming the work of Songsorn et al. [21], the results of the present study suggest that REHIT avoids large negative peaks of displeasure. Diminishing pleasure was observed during later exercise sessions as the intervention progressed. and these decreases in FS were probably associated with the increased number of sprints per session, which was necessary to achieve progressive overload. Nevertheless, average FS responses did not enter the negative domain at any time point, suggesting the protocols were well tolerated. Furthermore, the decreased sprint duration of SSREHIT resulted in more pleasurable FS responses at all time points compared to TREHIT suggesting this protocol could be deemed more manageable for some people which might be important when developing strategies for delivering REHIT in real-world settings. Affective valence is an important part of the exercise experience because pleasure-displeasure responses may predict adherence. This is based on the dual-mode theory which is predicated on the notion that exercise intensities exceeding ventilatory threshold are associated with unanimous feelings of displeasure [17, 20]. More negative affective responses have been observed for higher volume HIT $[17,36]$ and continuous vigorous exercise [36] but our data suggest REHIT is not associated with such negative affective responses, validating the findings of others [21, 22]. 
RPE scores recorded for TREHIT were higher than previously described by others $[11,12]$. These differences could be explained by variations in participant exercise history or baseline fitness, but more likely signify the different times at which these values were recorded. Metcalfe et al. $[11,12]$ asked participants to retrospectively consider effort for the whole training session after exercise cessation, whereas in the present study values were intentionally recorded shortly after sprints during exercise. For future research, it will be important to capture a representative depiction of cognitive responses during activity because changes in affect and perceived exertion may occur during exercise but then dissipate before post-exercise measurements are recorded, or may be hidden by the general shift in affect toward pleasure after exercise cessation [20]. These exhortations on the timing of measurement are crucial, as they are potentially decisive motivational factors for future exercise adherence with REHIT $[17,20]$. Furthermore, exercise protocols that elicit excessively high RPE responses are likely to be intolerable for the general population and would pose a significant barrier for most people. Although the volume-intensity relationship for REHIT could be considered ad infinitum, future iterations of REHIT should focus on the optimal combination of sprint frequency, sprint duration, and sprint intensity to induce health benefits in a practical, time-efficient, and tolerable manner without overly compromising perceptual responses.

Participants considered both REHIT conditions to be enjoyable. However, despite the differences in FS and RPE responses, there were no differences in enjoyment between conditions recorded 5-min after cessation of activity. Although affect and enjoyment overlap, they are not identical constructs, both of which are important in understanding the exercise experience. Enjoyment is an emotional experience requiring cognitive appraisal and appreciation of the totality of the exercise experience, whereas perceptions of affect pertain to rudimentary sensations of pleasure or pain [37] and are therefore more likely to correspond with homeostatic disturbances caused by relatively high exercise intensities. Nevertheless, because TREHIT was perceived to be more strenuous than SSREHIT, the findings of the current study support previous research findings [38] that higher-intensity exercise can be perceived to be as enjoyable as less-intense exercise in recreationally active men. Despite this, enjoyment diminished in both exercise conditions over time corresponding with reductions in affect as the exercise intervention progressed. The reason for this reduction in enjoyment remains unclear if it was not caused 
by the gradual increase in exercise intensity. However, it has been suggested that the nature of intermittent exercise can provide a succession of positive accomplishments as sprints are completed, and that this also breaks the activity into distinct parts which could make the activity appear more manageable preventing monotony [39]. A plausible explanation for the reduction in enjoyment therefore might be that the cognitive responses to REHIT diminished over time as the novelty of the exercise was reduced. Research to date has generally observed favourable enjoyment responses to HIT and REHIT [36, 39, 40], but has not considered how these perceptions change over time. Thus, a novel finding of our research is that perceptual responses may diminish over time, and that further longitudinal research is required to consider responses to REHIT in the longer-term.

The results of this study suggest that SSREHIT could be used as a time-efficient, yet tolerable, and adequately enjoyable approach to improve aerobic fitness. However, there are limitations to this protocol which should be considered. It was necessary to counter reductions in duration of sprints by increasing the frequency of sprints per session. This significantly reduces rest between sprints and could adversely disturb affect and RPE, although this was not evident in the present study. Furthermore, it is possible that increasing the number of sprints could result in 'pacing' strategies that may change the 'all-out' nature of the sprints which might be necessary for optimal adaptation [10]. Finally, the current study was undertaken in a controlled laboratory environment and participants received verbal encouragement to work maximally during each sprint, but effort may diminish for later sprints in unsupervised real-world settings. The need to carefully balance the frequency, duration, and intensity of sprints is a challenging issue.

Several sample and research design limitations should be considered alongside the findings of this study. Participants were young, healthy adults limiting the applicability of the findings to wider populations, particularly those who are inactive or who have chronic disease. Also, to capture a valid depiction of perceptual responses, measurements were taken at standardised time points throughout each condition, but since each condition used differing sprint durations and frequencies, these standardised time points represented marginally different parts of the exercise experience. However, this concern can be mitigated by the fact that peak perceptual values for each condition were recorded in close temporal proximity to sprints, thus physiological responses were unlikely to have dissipated 
before outcomes were recorded. Again, previous research [e.g. 11, 12, 18] has typically used three sessions of REHIT per week, whereas in the present study participants could choose to complete two or three sessions per week. This limits comparison between studies, although other research [40] has demonstrated that changes in maximal aerobic capacity are not significantly different when undertaking two or three sessions per week, and a strength of our design is improved ecological validity because participants had more autonomy and flexibility as they would in a real-world setting. This might have helped achieve the observed high intervention adherence, which alongside reporting of minimal adverse events and no participant drop out are further strengths of the study.

\section{Conclusion}

These data show that both REHIT conditions improved $\dot{V} \mathrm{O}_{2 \text { peak }}$ whilst minimising negative affective responses, although physiological adaptations might be attenuated with shorter duration sprints. SSREHIT resulted in more favourable pleasure and perceived exertion responses, so provided additional benefit in this regard. Future research should investigate the optimal dose-response relationship to elucidate the minimal amount of exercise to improve health outcomes in people with chronic diseases and reduce the burden of physical inactivity, as well as monitoring engagement and adherence to these protocols in the free living.

\section{Declaration of interest statement}

The authors have no conflicts of interest to report.

\section{Funding}

This research did not receive any specific grant from funding agencies in the public, commercial, or not-for-profit sectors. 


\section{References}

1. Warburton DER, Bredin SSD. Health Benefits of Physical Activity: A Systematic Review of Current Systematic Reviews. Curr Opin Cardiol 2017; 32(5): 541-556.

2. Reichart FF, Barros AJD, Dominigues MR et al. The role of perceived personal barriers to engagement in leisure-time physical activity. Am J Public Health 2007; 97: 515-519.

3. Hallal PC, Anderson LB, Bull FC et al. Physical Activity Series Working Group: Global physical activity levels: surveillance progress, pitfalls and prospects. Lancet 2012; 380: 247-257.

4. Haskell W, Lee I, Pate R et al. Physical Activity and Public Health: Updated Recommendation for Adults from the American College of Sports Medicine and the American Heart Association. Med Sci Sports Exerc 2007; 39(8): 1423-1434.

5. Department of Health and Social Care, UK. Physical activity guidelines: UK Chief Medical Officers' report. 2019. https://www.gov.uk/government/publications/physical-activity-guidelines-uk-chief-medical-officersreport. Accessed 09 Jul 2020.

6. Gaeser AG, Angadi S. High-intensity interval training for health and fitness: can less be more? J App Physiol 2011; 111: 1540-1541.

7. Gillen JB, Gibala MJ. Is high-intensity interval training a time-efficient exercise strategy to improve health and fitness? Appl Physiol Nutr Metab 2014; 39(3): 409-412.

8. Batacan RB, Duncan MJ, Dalbo VJ et al. Effects of high-intensity interval training on cardiometabolic health: a systematic review and meta-analysis of intervention studies. Br J Sports Med 2017; 51(6): 494-503.

9. Hardcastle SJ, Ray H, Beale L et al. Why sprint interval training is inappropriate for a largely sedentary population. Front Psychol 2014. 5: 1505. doi: 10.3389/fpsyg.2014.01505

10. Vollard NBJ, Metcalfe RS. Research into the health benefits of sprint interval training should focus on protocols with fewer and shorter sprints. Sports Med 2017. doi: 10.1007/s40279-017-0727-X

11. Metcalfe R, Babraj J, Fawkner S et al. Towards the minimal amount of exercise for improving metabolic health: beneficial effects of reduced-exertion high-intensity interval training. Eur J Appl Physiol 2011. 112: 2767-2775.

12. Metcalfe RS, Tardif N, Thompson D et al. Changes in aerobic capacity and glycaemic control in response to reduced-exertion high-intensity interval training (REHIT) are not different between sedentary men and women. Appl Physiol Nutr Metab 2016; 41(11): 1117-1123. 
13. Matsuo T, Saotome K, Seino S et al. Effects of a low-volume aerobic-type interval exercise on VO2max and cardiac mass. Med Sci Sports Exerc 2014. 46(1): 42-50.

14. Church TS, LaMonte MJ, Barlow CE et al. Cardiorespiratory fitness and body mass index as predictors of cardiovascular disease mortality among men with diabetes. Arch Intern Med 2005; 165(18): 2114-2120.

15. Ross R, Blair SN, Arena R et al. Importance of Assessing Cardiorespiratory Fitness in Clinical Practice: A case for fitness as a clinical vital sign: A scientific statement from the American Heart Association. Circ 2016; 134(24): e653-e699.

16. Ekkekakis P, Hall EE, Petruzzello SJ. Variation and homogeneity in affective responses to physical activity of varying intensities: An alternative perspective on dose-response based evolutionary considerations. J Sports Sci 2005; 23: 477-500.

17. Decker ES, Ekkekakis P. More efficient perhaps, but at what price? Pleasure and enjoyment responses to high-intensity interval exercise in low-active women with obesity. Psychol Sport Exerc 2017; 28: 1-10.

18. Ruffino JS, Songsorn P, Haggett M et al. A comparison of the health benefits of reduced-exertion highintensity interval training (REHIT) and moderate-intensity walking in type 2 diabetes patients. Appl Physiol Nutr Metab 2016; 10.1139/apnm-2016-0497.

19. Haines M. Assessing the feasibility of a reduced exertion, low-volume, high-intensity interval training (HIT) protocol: a pilot study. J Sports Sci 2015; 33(S1): S25-S31.

20. Ekkekakis P, Hall EE, Petruzzello SJ. The relationship between exercise intensity and affective responses demystified: To crack the 40-year-old nut, replace the 40-year-old nutcracker! Ann Behav Med 2008; 35: $136-149$.

21. Songsorn P, Fitzpatrick B, McDermott G et al. Affective and Perceptual Responses during ReducedExertion High-Intensity Interval Training (REHIT). Int J Sport Exerc Psych 2019; doi: 10.1080/1612197X.2019.1593217

22. Nalçakan GR, Songsorn P, Fitzpatrick BL, et al. Decreasing sprint duration from 20 to $10 \mathrm{~s}$ during reducedexertion high-intensity interval training (REHIT) attenuated the increase in maximal aerobic capacity but has no effect on affective and perceptual responses. Appl Physiol Nutr Metab 2019; 43(4): 338-344.

23. Metcalfe RS, Koumanov F, Ruffino JS et al. Physiological and molecular responses to an acute bout of reduced-exertion high-intensity interval training (REHIT). Eur J Appl Physiol 2015; 115(11): 2321-2334.

24. Haines M, Broom D, Gillibrand W et al. Effects of three low-volume, high-intensity exercise conditions on affective valence. J Sports Sci 2019; doi: 10.1080/02640414.2019.1684779

25. Cohen, J. Statistical power for behavioural sciences. Hillsdale: Lawrence Erlbaum Associates, 1988. 
26. Harriss DJ, Macsween A, Atkinson G. Ethical Standards in Sport and Exercise Science Research: 2020 Update. Int J Sports Med 2019; 40: 813-817

27. Hardy CJ, Rejeski WJ. Not what, but how one feels: The measurement of affect during exercise. J Sports Exerc Psychol 1989; 11: 304-317.

28. Borg G. Perceived exertion as an indicator of somatic stress. Scand J Rehabil Med 1970; 2: 92-98.

29. Stanley DM, Cumming. Are we having fun yet? Testing the effects of imagery use on the affective and enjoyment responses to acute moderate exercise. Psychol Sport Exerc 2009; 11: 582-590.

30. Milanović Z, Sporiš G, Weston M. Effectiveness of High-Intensity Interval Training (HIT) and Continuous Endurance Training for $\mathrm{VO}_{2 \max }$ Improvements: A Systematic Review and Meta-Analysis of Controlled Trials. Sports Med 2015; 45(10): 1469-1481.

31. Myers J, Prakash M, Froelicher V et al. Exercise capacity and mortality among men referred for exercise testing. N Engl J Med 2002; 346(11): 793-801.

32. Gurd BJ, Giles MD, Bonafiglia JT et al. Incidence of Non-Response and Individual Patterns of Response Following Sprint Interval Training. Appl Physiol Nutr Metab 2016; doi: 10.1139/apnm-2015-0449

33. Bouchard C, An P, Rice T et al. Familial aggregation of VO2max response to exercise training: results from the HERITAGE Family Study. J Appl Physiol 1999; 87(3): 1003-1008.

34. Timmons JA, Knudsen S, Rankinen T et al. Using molecular classification to predict gains in maximal aerobic capacity following endurance exercise training in humans. J Appl Physiol 2010; 108(6): 1487-1496.

35. Bonafiglia JT, Rotundo MP, Whittall JP et al. Inter-Individual Variability in the Adaptive Responses to Endurance and Sprint Interval Training: A Randomized Crossover Study. PLoS One 2016; 11(12): e0167790. doi:10.1371/journal.pone.0167790

36. Jung ME, Bourne JE, Little JP. Where does HIT fit? An examination of the affective response to highintensity intervals in comparison to continuous moderate- and continuous-vigorous intensity exercise in the exercise intensity-affect continuum. PLoS One 2014; 9(12): doi:10.1371/journal.pone.0114541

37. Wankel LM. The importance of enjoyment to adherence and psychological benefits from physical activity. Int J Sport Psychol 1993; 24: 151-169.

38. Bartlett J, Close G, MacLaren D et al. High-intensity interval running is perceived to be more enjoyable than moderate-intensity continuous exercise: Implications for exercise adherence. J Sport Sci 2011; 29: 547-53.

39. Martinez N, Kilpatrick MW, Salomon K et al. Affective and enjoyment responses to high-intensity interval training in overweight-to-obese and insufficiently active adults 2014; J Sport Exerc Psychol, 37(2), 138-149. 
40. Thomas G, Songsorn P, Gorman A et al. Reducing training frequency from 3 or 4 sessions/week to 2 sessions/week does not attenuate improvements in maximal aerobic capacity with reduced-exertion highintensity interval training (REHIT). Appl Physiol Nutr Metab 2020; 54(6): 683-685. 
Fig. 1. Schematic overview of both exercise conditions: a) SSREHIT, and b) TREHIT. For SSREHIT, all sprints were of 5-s duration. For TREHIT, the duration of sprints increased from 10-s for sessions 1 to 3 , to 15 -s for sessions 4 to 7 , and 20-s for sessions 8 to 15. Abbreviations: FS = Feeling Scale; RPE $=$ rating of perceived exertion; SSREHIT $=$ shortened-sprint, reduced-exertion high-intensity interval training; TREHIT = traditional reduced-exertion, high-intensity interval training.

Fig. 2. The change in $\dot{V} \mathrm{O}_{2 \text { peak }}$ following 15 sessions of SSREHIT and TREHIT. Abbreviations: SSREHIT $=$ shortened-sprint, reduced-exertion, high-intensity interval training; TREHIT = traditional reduced-exertion, high-intensity interval training; $\dot{V} \mathrm{O}_{2 \text { peak }}=$ peak oxygen uptake. Data are presented as mean \pm standard deviation. Closed circles denote individual responses. * Statistically significant in comparison to baseline value $(p \leq 0.01)$.

Fig. 3. Comparison of exercise conditions for a) peak FS, b) peak RPE, and c) enjoyment responses across the intervention. FS and RPE were recorded at $75 \%$ of bout completion. EES was recorded 5min after completion of exercise. Abbreviations: EES = Exercise Enjoyment Scale; FS = Feeling Scale; RPE = Rating of Perceived Exertion; SSREHIT = shortened-sprint, reduced-exertion high-intensity interval training; TREHIT $=$ traditional reduced-exertion, high-intensity interval training. Data are presented as aggregate mean $\pm 95 \%$ confidence intervals. $*$ Significant difference between groups $(p \leq$ $0.01)$. ** Significant difference between groups $(p \leq 0.001)$. 

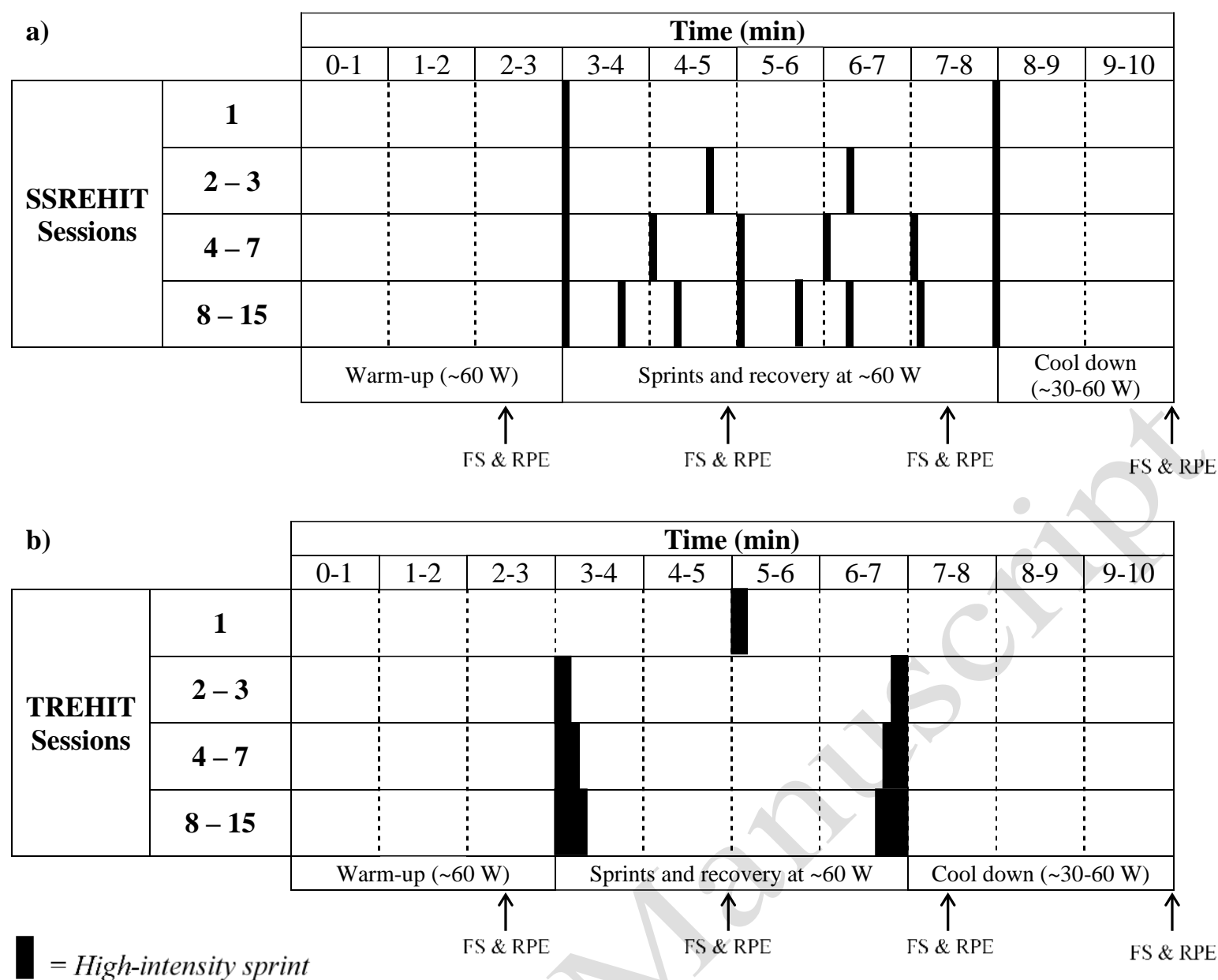


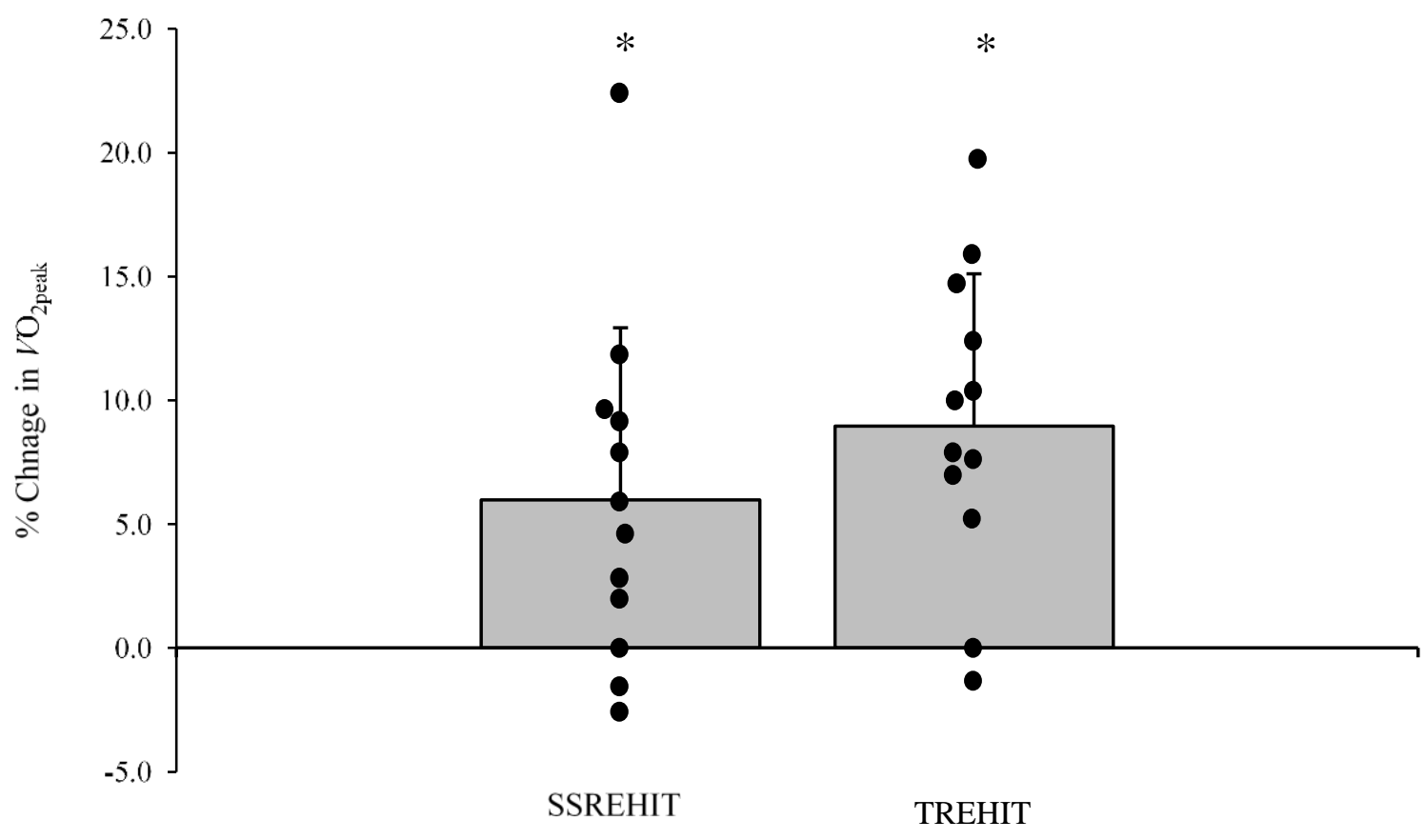


a)

$\multimap$ SSREHIT $\quad-$ TREHIT

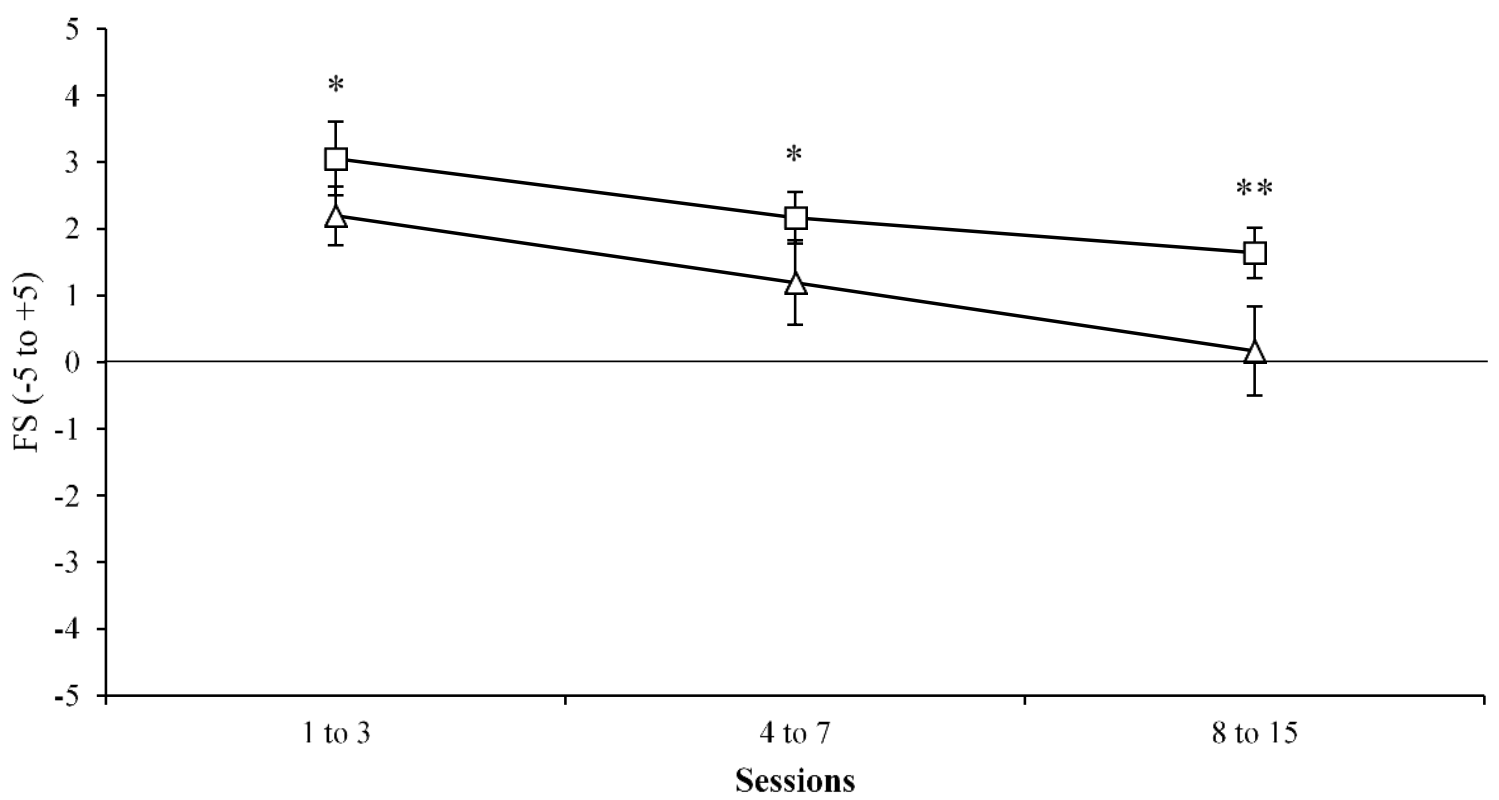

b)

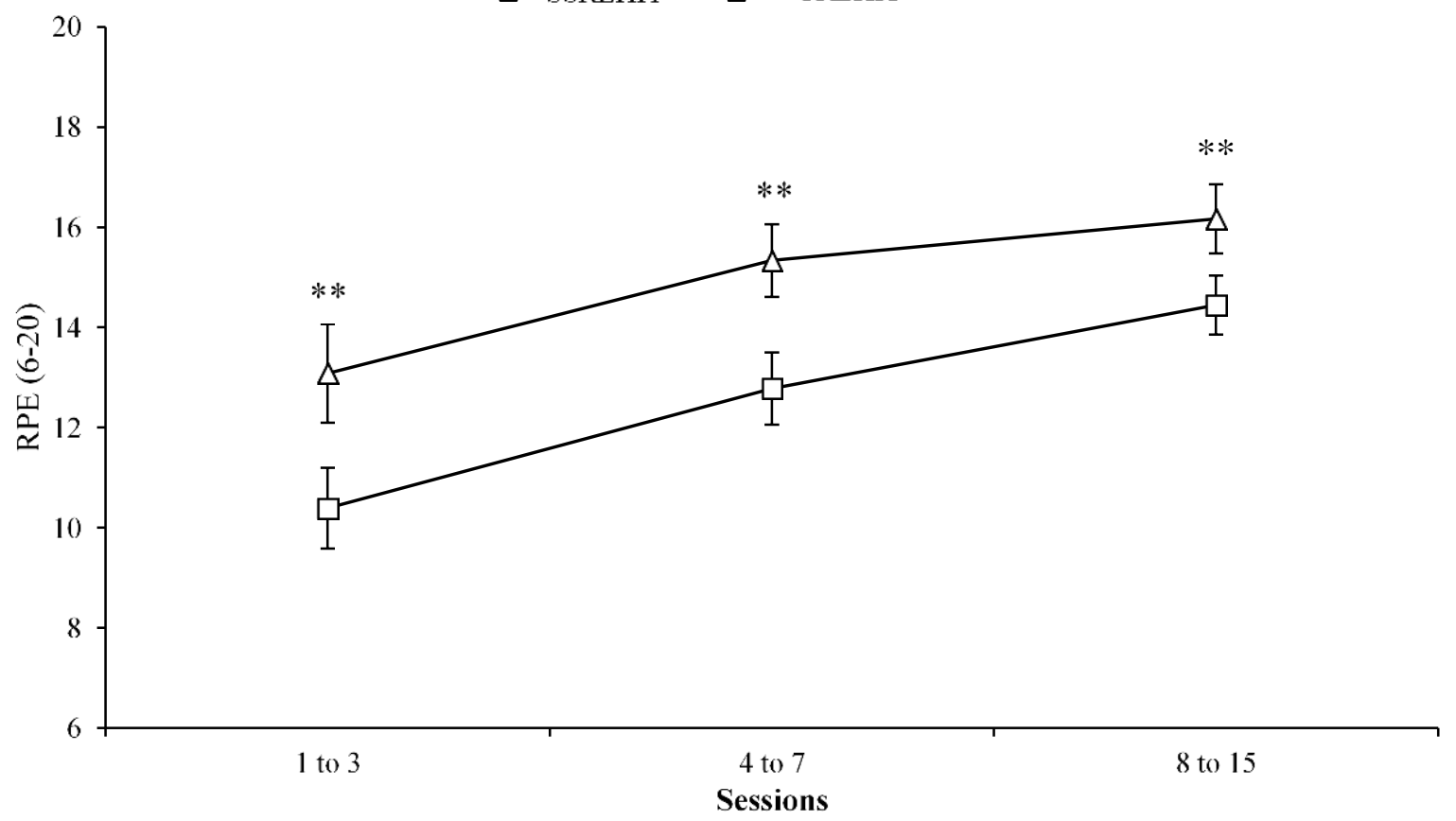




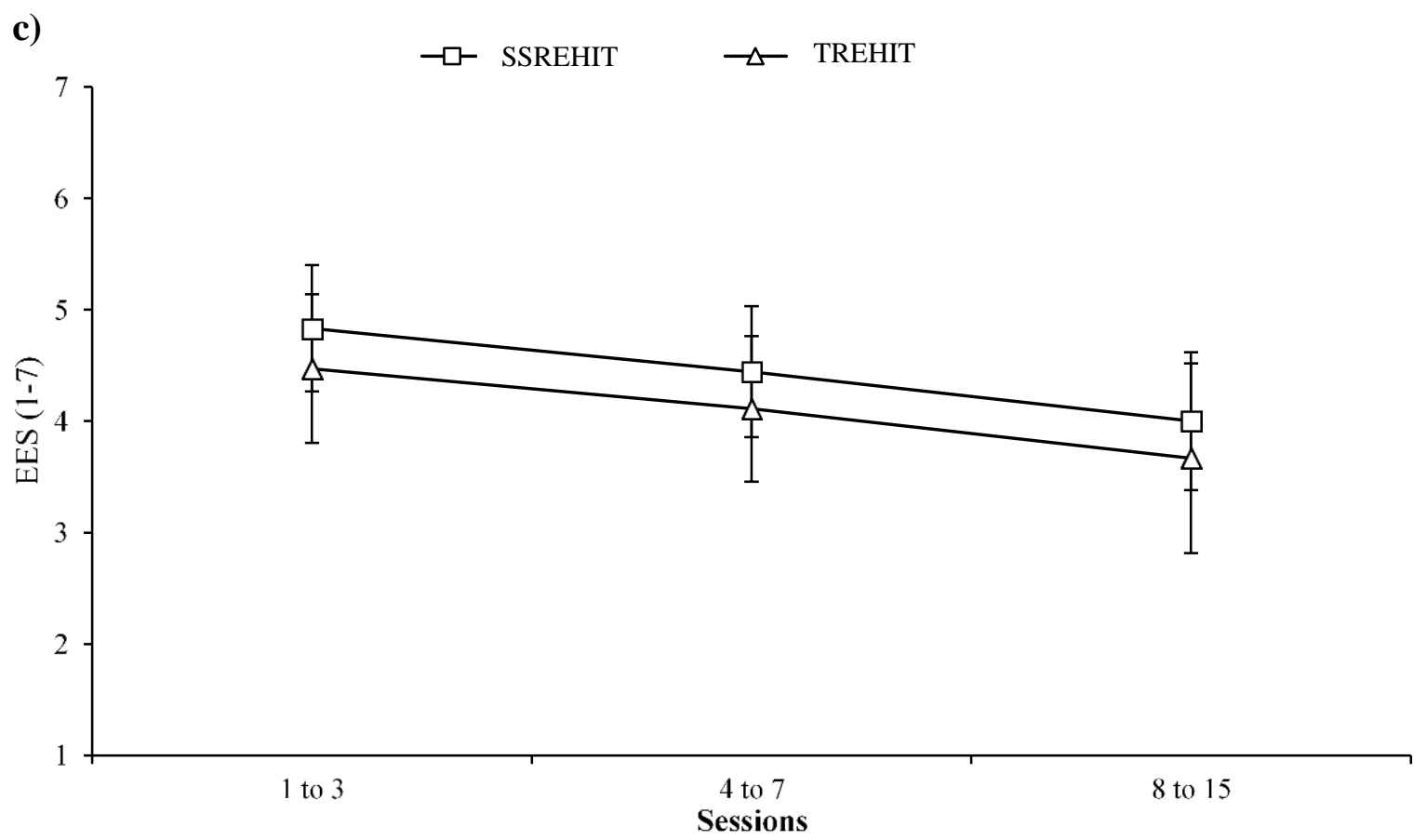


Table 1. Summary of responses for both exercise conditions.

\begin{tabular}{|c|c|c|c|c|}
\hline & \multirow[t]{2}{*}{ SSREHIT } & \multirow[t]{2}{*}{ TREHIT } & \multicolumn{2}{|c|}{ SSREHIT vs TREHIT } \\
\hline & & & $p=$ & $d=$ \\
\hline \multicolumn{5}{|l|}{$\underline{\mathbf{F S}}$} \\
\hline Sessions $1-3$ & $3.1 \pm 0.9^{*}$ & $2.2 \pm 0.7$ & 0.01 & 1.06 \\
\hline Sessions 4-7 & $2.2 \pm 0.6^{*}$ & $1.2 \pm 1$ & 0.01 & 1.16 \\
\hline Sessions 8-15 & $1.6 \pm 0.6^{*}$ & $0.2 \pm 1$ & 0.001 & 1.62 \\
\hline Average & $2.3 \pm 0.7$ & $1.2 \pm 1$ & - & - \\
\hline \multicolumn{5}{|l|}{ RPE } \\
\hline Sessions $1-3$ & $10.4 \pm 1.3 *$ & $13.1 \pm 1.6$ & 0.001 & -1.77 \\
\hline Sessions 4-7 & $12.8 \pm 1.1^{*}$ & $15.3 \pm 1.1$ & 0.001 & -2.17 \\
\hline Sessions $8-15$ & $14.4 \pm 0.9^{*}$ & $16.2 \pm 1.1$ & 0.001 & -1.71 \\
\hline Average & $12.5 \pm 2$ & $14.9 \pm 1.6$ & - & - \\
\hline \multicolumn{5}{|l|}{ EES } \\
\hline Sessions 1-3 & $4.8 \pm 0.9$ & $4.5 \pm 1$ & 0.37 & 0.30 \\
\hline Sessions 4-7 & $4.4 \pm 0.9$ & $4.1 \pm 1$ & 0.41 & 0.30 \\
\hline Sessions $8-15$ & $4 \pm 1$ & $3.7 \pm 1.3$ & 0.49 & 0.25 \\
\hline Average & $4.4 \pm 0.4$ & $4.1 \pm 0.4$ & - & - \\
\hline
\end{tabular}

